\title{
BMJ Open Effects of novel flash glucose monitoring system on glycaemic control in adult patients with type 1 diabetes mellitus: protocol of a multicentre randomised controlled trial
}

\author{
Yongwen Zhou (D) , ${ }^{1,2}$ Hongrong Deng, ${ }^{2}$ Hongxia Liu, ${ }^{2}$ Daizhi Yang, ${ }^{2}$ Wen $\mathrm{Xu},{ }^{2}$ \\ Bin Yao, ${ }^{2}$ Jinhua Yan, ${ }^{2}$ Jianping Weng ${ }^{1}$
}

To cite: Zhou Y, Deng $\mathrm{H}$, Liu $\mathrm{H}$, et al. Effects of novel flash glucose monitoring system on glycaemic control in adult patients with type 1 diabetes mellitus: protocol of a multicentre randomised controlled trial. BMJ Open 2020;10:e039400. doi:10.1136/ bmjopen-2020-039400

- Prepublication history and additional materials for this paper is available online. To view these files, please visit the journal online (http://dx.doi. org/10.1136/bmjopen-2020039400).

YZ and HD contributed equally.

Received 15 April 2020

Revised 16 0ctober 2020 Accepted 03 November 2020

Check for updates

(C) Author(s) (or their employer(s)) 2020. Re-use permitted under CC BY-NC. No commercial re-use. See rights and permissions. Published by BMJ.

For numbered affiliations see end of article.

Correspondence to Dr Jinhua Yan; yanjh79@163.com and Dr Jianping Weng; wengjp@ustc.edu.cn

\section{ABSTRACT}

Introduction Optimal glycaemic control is beneficial to prevent and delay microvascular complications in patients with type 1 diabetes mellitus (T1DM). The benefits of flash glucose monitoring (FGM) have been proved among well-controlled adults with T1DM, but evidence for FGM in adults with T1DM who have suboptimal glycaemic control is limited. This study aims to evaluate the effect of FGM in suboptimally controlled adult patients with T1DM .

Methods and analysis This open-label, multicentre, randomised trial will be conducted at eight tertiary hospitals and recruit 104 adult participants $(\geq 18$ years old) with T1DM diagnosed for at least 1 year and with suboptimal glycaemic control (glycated haemoglobin (HbA1c) ranging from $7.0 \%$ to $10.0 \%$ ). After a run-in period (baseline, $0-2$ weeks), eligible participants will be randomised 1:1 to either use FGM or self-monitoring of blood glucose alone consequently for the next 24 weeks. At baseline, 12-14 weeks and 24-26 weeks, retrospective continuous glucose monitoring (CGM) systems will be used in both groups for device-related data collection. Biological metrics, including $\mathrm{HbA1c}$, blood routine, lipid profiles, liver enzymes, questionnaires and adverse events, will be assessed at baseline, week 14 and week 26 . All analyses will be conducted on the intent-to-treat population. Efficacy endpoint analyses will also be repeated on the per-protocol population. The primary outcome is the change of $\mathrm{HbA} 1 \mathrm{c}$ from baseline to week 26 . The secondary outcomes are the changes of CGM metrics, including time spent in range, time spent in target, time spent below range, time spent above range, SD, coefficient of variation, mean amplitude of glucose excursions, high or low blood glucose index, mean of daily differences, percentage of $\mathrm{HbA} 1 \mathrm{c}$ in target $(<7 \%)$, frequency of FGM use, total daily insulin dose and the scores of questionnaires including Diabetes Distress Scale, Hypoglycemia Fear Scale and European Quality of Life Scale.

Ethics and dissemination This study was approved by the Ethics Committee of the Third Affiliated Hospital of Sun Yat-sen University in January 2017. Ethical approval has been obtained at all centres. All participants will be provided with oral and written information about the
Strengths and limitations of this study

- This study adopts a multicentre, open-label, randomised and parallel design.

- This study aims to evaluate the flash glucose monitoring system among adult patients with type 1 diabetes mellitus who have suboptimal glycaemic control with the comparison with self-monitoring of blood glucose.

- The retrospective continuous glucose monitoring (CGM) system will provide detailed comparative data on efficacy and safety between the two study arms.

- There is a head-to-head comparison on the sensorrelated metrics as patients randomised to use the flash glucose monitoring systems will wear the retrospective CGM systems additionally and simultaneously in the 14 days preceding the 3 and 6-month visiting.

- The limitation of this study is that the questionnaires evaluating the satisfaction with the device are not used in this trial.

trial. The study will be disseminated by peer-review publications and conference presentations. Trial registration number NCT03522870.

\section{INTRODUCTION}

The Diabetes Control and Complications Trial had demonstrated that intensive glycaemic control contributes to delay and prevents the development and progression of microvascular complications. ${ }^{1}$ However, even with much advancement of diabetes management in these years, such as the improvement of insulin analogues and insulin infusion pumps, it is still difficult for adult patients with type 1 diabetes mellitus (T1DM) to achieve the recommended goals of HbAlc level $(<7 \%)$ and the target-achieving rate was only approximately $15 \%-30 \% .^{2-6}$ As glucose 
monitoring is one of the key parts of diabetes management and previous studies had demonstrated a strong association between glucose monitoring and glycaemic control in patients with $\mathrm{T} 1 \mathrm{DM},{ }^{57}$ the optimisation of glucose monitoring is necessary.

The conventional glycaemic monitoring methods include the daily self-monitoring of blood glucose (SMBG) by fingerstick tests and HbA1c tests. The SMBG is the most widely used glucose testing method and generally enjoys good accuracy whereas it only provides the single point-in-time glucose concentrations instead of overall daily profiles and the pain from fingerstick might lead to decrease of the participants' adherence. The HbA1c, the golden standard of glycaemic monitoring method, reflecting the average glucose concentration for approximately 3 months, is also not direct and convenient enough for not proving a measure of glycaemic variability or an alert function of real-time hypoglycaemia moments. ${ }^{6}$ Therefore, an alternative of the glucose monitoring method in recent years is the updated continuous glucose monitoring (CGM) technology, which provides near realtime glucose data continuously by tracking the glucose concentrations in the body's interstitial fluid and reflects the intraday/interday glycaemic excursions. There are two basic types of CGMs. One is the retrospective CGM with blinded data available to users and clinicians, which is usually applied in the outpatient visits or clinical trials. The other one is the systems that provide unblinded data such as the real-time CGM systems. It has been demonstrated that glycaemic control and psychological status of the adult patient with T1DM can be improved after using the real-time CGMs, ${ }^{8-10}$ and the benefits can also be sustained for 12 months when used properly. ${ }^{11}$

For most CGMs, SMBG is still required for calibrations. While the flash glucose monitoring (FGM) system (FreeStyle Libre; Abbott Diabetes Care, Witney, Oxon, UK), the new generation of CGMs, approved by Food and Drug Administration (FDA) in 2017, is factory calibrated and provides a longer sensor lifetime of 14 days, which has further relieved the pain from frequent strip capillary glucose calibrations and thus is relatively more acceptable and easier for widespread use. To date, most relevant published articles were research regarding the accuracy of $\mathrm{FGM}^{12-14}$ and reviews discussing its clinical effectiveness, cost-effectiveness and safety, ${ }^{15-17}$ while there were only a small number of randomised clinical trials (RCTs) and protocols available to prove its benefits in patients with T1DM. ${ }^{18-22}$ Although data from these trials are encouraging, it remains unclear whether the FGM is effective in adult patients with T1DM who had suboptimal glycaemic control. Therefore, we designed this 24-week comparative trial, aiming to evaluate the effect of FGM in adult patients with T1DM who have suboptimal glycaemic control. The research protocol of the RCT study is presented below.

\section{METHODS AND ANALYSIS \\ Study design}

This trial is an open-label, multicentre, randomised and parallel-group study conducted at eight centres in seven cities (Guangzhou, Hefei, Foshan, Zhongshan, Shanghai, Wuhan and Shenzhen) in China. Eligible participants will be recruited and the efficacy of FGM and SMBG in adult patients with T1DM who have suboptimal glycaemic control will be compared. Written informed consent will be obtained from all participants before study-related activities (see online supplemental file 1). This trial has been approved by the Ethics Committee of the Third Affiliated Hospital of Sun Yat-sen University and conformed to the Declaration of Helsinki.

\section{Study procedure}

The flow chart of this study is presented in figure 1 . After a run-in period of 2 weeks, eligible participants will be randomised 1:1 to either use of FGM or SMBG consequently for 24 weeks. At baseline (0-2 weeks), 12-14 weeks and 24-26 weeks, retrospective CGMs (Ipro2) will be additionally used in both groups. Demographic and biological data, questionnaires and adverse events (AE) will also be collected and assessed at baseline, week 14 and week 26.

\section{Participant recruitment (before 0 week)}

The recruitment has begun in May 2018 and will extend to December 2021. Major eligibility criteria include age $\geq 18$ years old, HbAlc between $7 \%$ and $10 \%$ and duration of T1DM at least 1 year. The diagnostic criteria of T1DM are based on the definition of T1DM by the American

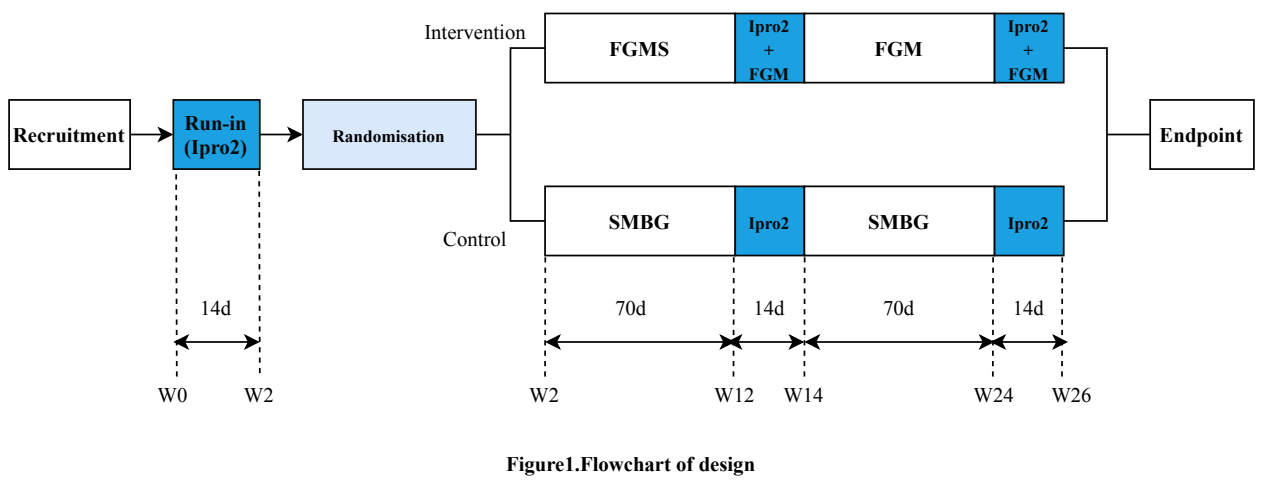

Figure 1 Flow chart of the design. FGM, flash glucose monitoring; SMBG, self-monitoring of blood glucose. 


\section{Box 1 Inclusive and exclusive criteria}

Inclusive criteria

Aged $\geq 18$ years

- Diagnosed with T1DM with the criteria established by WHO in 1999, and with duration more than 1 year.

- Glycosylated haemoglobin A1c concentration between $7 \%$ and $10 \%$.

- SMBG daily ( $\geq 3$ times per day) for at least 2 months prior to study entry and willing to insist for at least 6 months.

- Stable insulin regimen medication including CSII and MDI for 3 months prior to study entry (change of insulin $\leq 20 \%$ ), not including premixed insulin.

- Willing to wear CGM.

Able to speak, read and write Chinese.

Exclusive criteria

- Having used any CGM 3 months prior to study entry.

- Receiving oral steroid therapy for any disorders and continuous use of paracetamol.

- Had known allergy to medical grade adhesives or CGM and its affiliated components.

- Being pregnant or planning pregnancy (as demonstrated by a positive test at study entry).

- Recent severe diseases like myocardial infarction, stroke, psychiatric diseases (historical/recent), malignant tumour, kidney disease (defined as estimated glomerular filtration rate $<45 \mathrm{~mL} / \mathrm{min} / 1.73$ $\mathrm{m}^{2}$ ) and dermatosis, decided by the investigator.

- Currently participating in another research (must have completed any study at least 30 days prior to being enrolled in this study).

- Currently abusing illicit drugs, alcohol or prescription drugs.

- Any condition that could impact reliability of $\mathrm{HbA1c}$ measurement, such as haemoglobinopathy, haemolytic anaemia and chronic liver disease, decided by the investigator.

CGM, continuous glucose monitoring; CSII, continuous subcutaneous insulin infusion; MDI, multiple daily injections; SMBG, self-monitoring of blood glucose; T1DM, type 1 diabetes mellitus.

Diabetes Association and the WHO. ${ }^{23}{ }^{24}$ Other inclusive and exclusive criteria are shown in box 1 .

Run-in period (baseline, weeks 0-2)

In this period, demographics, medical histories, smoking or drinking status, exercise and the results of physical examination (body mass index, the waist to hip ratio, blood pressure and heart rate) will be collected by certified physicians and nurses in accordance with standardised protocols. Urine samples will be collected for the measurements of albumin to creatine ratio and female participants will have extra urine pregnancy tests in the participant centres. Fasting blood samples are collected for biological measurements. Biological metrics including HbAlc, blood routine, lipid profiles, liver enzymes, thyroid function and antibodies, C-peptide, and diabetes antibodies will be tested centrally in the laboratory of the Third Affiliated Hospital of Sun Yat-sen University. In addition, questionnaires including the Chinese version of Diabetes Distress Scale, ${ }^{25}$ Hypoglycemia Fear Scale ${ }^{26}$ and European Quality of Life Scale ${ }^{27}$ will be completed by participants.

Then, all participants will wear the retrospective CGM (Ipro2, Medtronic, USA) on the back of the upper arms continuously for 2 weeks. Blood glucose metres and compatible test strips (Bayer; Bayer Consumer Care) will be distributed to all participants for capillary blood glucose tests during the whole study period and instructions about device use will be provided simultaneously. The detailed introduction of the questionnaires, the Ipro2 and the blood glucose metres will be presented in the online supplemental file 2. During 2 weeks, capillary blood glucose tests, diet diary and exercise will be required to record for calibration. Sensor glucose measurements will not be visible to the patients and the investigators until the data are downloaded via the CareLink iPro software after 2 weeks and then calculated by the Glyculator 2.0 software which follows the guidelines on CGM reporting specified in the international consensus on use of CGM. ${ }^{28}$ Participants in both groups will be instructed on the general diabetic education with standard algorithms including self-management suggestions for hypoglycaemia/hyperglycaemia and suggestions for insulin titration (see online supplemental file 3).

\section{Randomisation}

After the 2-week run-in period, eligible participants will be randomised 1:1 to either daily SMBG alone or FGM. The random sequence will be generated by SPSS 20.0 software and arranged into the sealed, opaque envelopes by investigators. To reduce the selection bias, there will be an independent researcher in charge of the envelope distribution only. When there is an eligible participant, the responsible investigator is required to inform the independent researcher. Then the sealed envelopes will be randomly distributed to the corresponding centre, where envelopes will be opened sequentially to determine the participants' assignments.

\section{Study intervention}

After randomisation, participants in the FGM group will be provided with FGM (FreeStyle Libre; Abbott Diabetes Care) and measure glucose concentrations at home for the following 24 weeks. Detailed introduction of FGM system will be presented in the online supplemental file 2 . Instructions about device use will be provided according to the manufacturer's user manual and access to the device software (FreeStyle Libre Software V.1.0; Abbott Diabetes Care) will be given. Participants will be required to report the AEs especially those relevant to the device such as the skin problems and the sensor early removal. An additional fingerstick test will be recommended for their decision-making when sensor data are below 3.9 or over $13.9 \mathrm{mmol} / \mathrm{L}$ but the frequency of the fingerstick tests is non-restricted. The first sensor will be applied by the trained staff and the rest will be applied by patients themselves every 2 weeks. The participants assigned to the SMBG group will be required to perform capillary glucose tests for at least three times per day during the following 6 months and record their daily glucose data. The additional fingerstick tests will be recommended 
when hypoglycaemia and hyperglycaemia-related symptoms occur in both groups.

Follow-up visits (weeks 12-14 and weeks 24-26)

Follow-up visits for both groups will be scheduled from week 12 to week 14 and from week 24 to week 26, during which professional CGM will be additionally used in both groups to collect CGM data for 2 weeks. During the 2-week follow-up, for both groups, data on fingerstick tests, diet, exercises and insulin adjustment during this period will be required to record for calibration but no extra education or suggestions on diabetic management will be provided by investigators until the end of the 2-week data collection. At the end of weeks 14 and 26 , glucose data collected from the Ipro2 during 2 weeks will be downloaded via the software and the sufficiency of sensor data during 2 weeks will also be assessed, ensuring at least $70 \%$ of data are available. Then, general diabetes education and insulin adjustment advice will be provided in both groups according to the standard algorithms and the ambulatory glucose profiles derived from the previous 2-week retrospective CGM wearing. Demographics and physical information, questionnaires and the biomedical samples will be collected at the same time.

For the FGM group, glucose data stored in the FGM recorders from week 2 to week 14 and from week 14 to week 26 will be downloaded respectively by research staff via its corresponding software. For the SMBG group, fingerstick glucose data stored in the blood glucose metres from week 2 to week 14 and from week 14 to week 26 will also be collected respectively.

\section{Endpoints}

The primary endpoint is the change in HbAlc levels from baseline to week 26. The major secondary endpoints include the change in time spent in range (3.9$10.0 \mathrm{mmol} / \mathrm{L})$, time spent in target $(3.9-7.8 \mathrm{mmol} / \mathrm{L})$, time spent below range (TBR $(<3.9 \mathrm{mmol} / \mathrm{L})$; TBR $(<3.0 \mathrm{mmol} / \mathrm{L}))$ and time spent above range (TAR $(>10.0 \mathrm{mmol} / \mathrm{L})$; TAR $(>13.9 \mathrm{mmol} / \mathrm{L}))$ from baseline to week 26, SD, coefficient of variation, mean amplitude of glucose excursions, high or low blood glucose index, mean of daily differences, percentage of $\mathrm{HbAlc}$ in the target $(<7 \%)$, frequency of FGM use, total daily insulin dose and the differences in scores of respective questionnaires. All predefined endpoints and the timing of all assessments are shown in table 1.

\section{Risks and AEs}

Once included, responsible investigators will trace if any device or study-related risks and AEs have occurred. Disease-related events that are chronic in nature and occur as part of the progression of the diabetes disease state (ie, diagnosis of retinopathy, nephropathy, neuropathy) will not be captured as AEs in this study.

As reported in the recent system reviews, ${ }^{29}$ the most common sensor wear-related cutaneous complication was erythema (55\%), followed by itching/pruritus (11\%),
Table 1 Endpoints

\begin{tabular}{|c|c|}
\hline \multicolumn{2}{|l|}{ Primary endpoints } \\
\hline HbA1c (\%) & $\begin{array}{l}\text { Difference in } \mathrm{HbA} 1 \mathrm{c} \text { at week } 26 \text { adjusted } \\
\text { for baseline }\end{array}$ \\
\hline \multicolumn{2}{|l|}{ Secondary endpoints } \\
\hline $\begin{array}{l}\text { CGM metrics* } \\
\text { (whole, night }(00: 00- \\
\text { 06:00), daytime } \\
(06: 00-00: 00))\end{array}$ & $\begin{array}{l}\text { The difference in CGM profiles listed below } \\
\text { collected via Ipro2 in week } 12-14 \text { and } \\
\text { week } 24-26 \text { adjusted for baseline (week } \\
0-2 \text { ) }\end{array}$ \\
\hline TIR (\%) & Range $3.9-10.0 \mathrm{mmol} / \mathrm{L}(70-180 \mathrm{mg} / \mathrm{dL})$ \\
\hline TIT (\%) & Range $3.9-7.8 \mathrm{mmol} / \mathrm{L}(70-140 \mathrm{mg} / \mathrm{dL})$ \\
\hline TBR (\%) & $\begin{array}{l}<3.9 \mathrm{mmol} / \mathrm{L}(70 \mathrm{mg} / \mathrm{dL}) ;<3.0 \mathrm{mmol} / \mathrm{L}(54 \\
\mathrm{mg} / \mathrm{dL})\end{array}$ \\
\hline TAR (\%) & $\begin{array}{l}>10 \mathrm{mmol} / \mathrm{L}(180 \mathrm{mg} / \mathrm{dL}) ;>13.9 \mathrm{mmol} / \mathrm{L} \\
(250 \mathrm{mg} / \mathrm{dL})\end{array}$ \\
\hline
\end{tabular}

Mean blood glucose (mmol/L)

Estimated A1c (\%)

SD

CV

MAGE

HBGI

LBGI

MODD

Number of

hypoglycaemia

events

- Percentage of $\mathrm{HbA1c}$ value in target (\%)

The difference in the percentage of $\mathrm{HbA} 1$ in range $(<7 \%)$ tested at week 14 and 26 adjusted for baseline

- Frequency of using Time frame: 24 weeks (from week 2 to FGM (times/day) $\dagger$

week 26)

Frequency of using Time frame: 24 weeks (from week 2 to SMBG (times/day) week 26)

- Total of daily insulin The difference in insulin dose collected at dose (IU/kg/day) week 14 and 26 adjusted for baseline

Questionnaires The difference in scores of respective questionnaires collected at week 14 and 26 adjusted for baseline

DDS

HFS

\section{EQ-5D-5L}

${ }^{*} \mathrm{CGM}$ metrics analysed here are calculated with the sensor data from Ipro2.

†The frequency of using FGM is calculated with the recordings derived from the FGM system.

CGM, continuous glucose monitoring; CV, coefficient of variation; DDS, Diabetes Distress Scale; EQ-5D-5L, European Quality of Life Scale; FGM, flash glucose monitoring; HBGl, high blood glucose index; HFS, Hypoglycemia Fear Scale; LBGI, low blood glucose index; MAGE, mean amplitude of glucose excursion; MODD, mean of daily differences; SMBG, self-monitoring of blood glucose; TAR, time above range; TBR, time below range; TIR, time spent in range; TIT, time spent in target.

induration $(9 \%)$, oedema (6.9\%), rash $(6.4 \%)$, bruising $(5.7 \%)$ and allergic reaction $(4.3 \%)$. The frequency of skin infection, dry skin, cellulitis and the collection was seldom reported with a percentage only from $0.2 \%$ 
to $0.7 \%$. The insertion of the sensor could also lead to cutaneous complications such as pain $(61.7 \%)$, bleeding $(37.6 \%)$ and haematoma $(0.7 \%)$. However, the incidence rate of these events is low with one event reported per 8 weeks of sensor wear time and the reported complication severity is also low with $78.6 \%$ rated as mild and only $1.5 \%$ rated as severe. Once these events occur, participants will be encouraged to consult for the responsible investigator. If there are no symptoms of infection or inflammations such as redness, swelling and aggravated pain, removal of the sensor is not recommended. After removal of the sensor, irritation might occur due to the medical adhesive, the bandages that may be placed over the device and the healing process, which is normal. This reaction is self-limiting and should resolve within hours.

Confirmed diabetes ketoacidosis, hyperosmolar hyperglycaemic state and severe hypoglycaemic events will be captured as serious AEs. According to the guidelines from the American Diabetes Association, ${ }^{6}$ the definition of severe hypoglycaemia is hypoglycaemia associated with severe cognitive impairment requiring external assistance for recovery. All study or device-related AEs will be monitored until adequately resolved or stable.

\section{Laboratory analyses and data management}

The HbAlc concentration is centrally measured by an automated analyser (Bio-Rad D10; Bio-Rad Laboratories, Hercules, California) using the high-performance liquid chromatography technique, with a reference range of $4.3 \%-6.1 \%$ and intrabatch and interbatch coefficients of variation of $0.46 \%$ and $0.99 \%$, respectively. Lipid profiles, liver enzymes and renal function are determined by the enzymatic colorimetric test with Hitachi 7600 autoanalyser. The thyroid function and its antibodies are assessed by the chemiluminescence immunoassay method using the ADVIA Centaur System (Siemens, Massachusetts, USA).

Fasting C-peptide is measured by an iodine $\left({ }^{125} \mathrm{I}\right)$ human C-peptide radioimmunoassay kit (Beijing North Institute of Biological Technology, Beijing, China; intrabatch and interbatch coefficients of variation of $0.46 \%$ and $0.99 \%$, respectively). Autoantibodies against the 65 $\mathrm{kDa}$ isoform of glutamic acid-decarboxylase antibody (GADA), insulinoma-associated protein-2 antibody (IA2A) and zinc transporter 8 autoantibody (ZnT8A) were analysed centrally using fasting serum with radiobinding assay confirmed by the Islet Autoantibody Standardization Program (assay sensitivity and specificity for GADA were $64 \%$ and $98 \%$, respectively; $64 \%$ and $100 \%$ for IA-2A, respectively; $36 \%$ and $98 \%$ for ZnT8A, respectively) at the First Affiliated Hospital of Nanjing University. Patients with positive results for at least one antibody titre tested (GADA titre $\geq 0.042$ was seen as positive; ZnT8A titre $\geq 0.054$ was seen as positive; IA-2A titre $\geq 0.018$ was seen as positive) were considered positive for diabetes autoantibodies.

The coordinator centre is located in the Third Affiliated Hospital of Sun Yat-sen University, Guangzhou,
China. Data in this trial including the demographics and non-centrally tested biological data will be collected using the case report forms by responsible participating investigators and sent to the coordinator centre periodically. To maintain the accessibility of the database, facilities will be conducted as follows: (1) All participating investigators will be trained before study commencement. Standardised procedures will be illustrated in detail. (2) The responsible associate investigators will monitor the data collection process and evaluate the data integrity periodically during the course of the data collection phase. (3) A secondary review of the accuracy of data recorded from all participating hospitals will be conducted by coauthors and the principal investigator will manage the data flow and perform audits of the procedure of the study.

\section{Sample size}

According to the RCTs about CGM, ${ }^{810} 30$ assuming a drop rate of $10 \%$, a sample size of 104 participants would be required for providing $80 \%$ power to detect a group difference in mean changes of HbAlc of $0.4 \%$ (SD 0.8 , correlation 0.6) using a two-sided test at the 0.05 level.

\section{Statistical analysis}

All analyses will be conducted on the intent-to-treat population. Data from all randomised patients with or without protocol violation including dropouts and withdrawals will be included in the analysis.

It is anticipated that subjects with T1DM who are suboptimally controlled will show an improvement in HbAlc level with the use of FGM in the intervention group after 24 weeks, over and above any improvement in subjects using SMBG in the control group. Changes in the primary and secondary outcomes will be analysed using a linear mixed model with management, time and their interaction as covariates. Change in outcome measures within each group and difference of the changes between groups from baseline to follow-up will be calculated using linear combinations of the estimated coefficients. If there are baseline imbalances between treatment groups, we will consider adjusting them based on whether we regard the imbalance as clinically significant. A $95 \%$ CI will be given for the difference between the groups.

The calculation of the CGM metrics during the whole time, the night period (00:00-06:00) and the daytime period (06:00-00:00) is via the Glyculator 2.0 software. Information including demographics and physical measurements will be summarised. The calculation of the questionnaires is presented in the online supplemental file 2. Continuous variables will be presented with mean \pm SD or median (25th and 75th quartile ranges). Categorical variables will be presented with the proportion of subjects in each category. If values are highly skewed, transformation or non-parametric analyses will be used. $X^{2}$ tests or Fisher's exact test will be used to analyse the categorical data. The safety analysis will include all available data from all recruited patients. Any device-related AEs will be tabulated and reported. All null 
hypotheses will be tested against a two-sided alternative at the $5 \%$ significance level.

\section{DISCUSSIONS}

The utilisation of CGM is increasing rapidly around the world. The benefits of the real-time CGM among adults, adolescents and elders with T1DM have been demonstrated previously. ${ }^{30-33}$ As a new category of CGM, the FGM remains interstitial data recorded every $15 \mathrm{~min}$ and special functions with no needs of SMBG calibrations, extended sensor spans and near real-time glucose value by scanning on demands. Several observational studies had demonstrated significant improvements in HbAlc with a change of $-0.55 \%$ after $2-4$ months of use. ${ }^{34}$ In the multicentre randomised controlled studies which was conducted either on well-controlled adult patients with T1DM or high-risk young adults (13-20years), the group using FGM showed insignificant improvements in HbAlc change while only those adults with well-controlled had reduced time spent in hypoglycaemia. ${ }^{18}{ }^{21}$ However, to date, there is still no evidence from RCTs conducted in adult patients with T1DM and suboptimal control. Different from the other CGMs, there is no hypoglycaemia alert function in FGM, which was thought to be less effective than real-time CGM system. ${ }^{19}$ Whether these patients who made up a large proportion of patients with T1DM would derive similar benefits from FGM or have similar compliance on FGM use is required to be discussed.

This trial will be conducted at eight centres that have an abundant experience in the treatment and management of T1DM. The trial will provide a 24-week consistent use of FGM in the intervention group, and collect the HbAlc value and 2-week CGM-related glycaemic metrics termly to compare their changes from baseline between FGM and SMBG. The result might provide a more comprehensive evaluation on clinical utility and reliability of the FGM in adults with T1DM under suboptimal glycaemic control.

There are some limitations to this trial. First, questionnaires evaluating the satisfaction with the devices are not used in this trial because there are no reliable Chinese versions of the scales until study commencement. Second, the period assessed in this trial is only for 6 months and the sustained effect of the FGM among patients with suboptimal glycaemic control assessed in the RCTs is required in the future.

\section{PATIENT AND PUBLIC INVOLVEMENT}

No patients were involved in the development of the research question or design of the study.

\section{ETHICS AND DISSEMINATION}

This trial wis conducted in accordance with the Declaration of Helsinki (1964) including all amendments and the 1983 amendment per FDA's Guidance for Industry. It was also approved by the Ethics Committee of the Third Affiliated Hospital of Sun Yat-sen University. Subjects will be provided the opportunity to review the informed consent before coming to the clinical site. The consenting process will be documented in the subject's source document.

\section{Author affiliations}

${ }^{1}$ Department of Endocrinology, the First Affiliated Hospital of USTC, Division of Life Science and Medicine, University of Science and Technology of China, Hefei, China ${ }^{2}$ Department of Endocrinology and Metabolism, Third Affiliated Hospital of Sun Yat-sen University; Guangdong Provincial Key Laboratory of Diabetology, Guangzhou, China

Contributors JW and JY designed and organised the study. YZ and HD registered the trial and cowrote the first draft of the manuscript. JW, JY and HL undertook a critical revision of the manuscript. YZ, HD and HL were responsible for the recruitment and implementation of the protocol. DY, WX and BY contributed to the data interpretation. JW and JY had full access to all the data in the study and had the final responsibility on the decision to submit for publication. All authors have read and approved the final manuscript.

Funding This study was supported by the National Key Research and Development Program of China (2017YFC1309600 to JW). All sensors will be purchased through this grant.

Disclaimer Bayer, Medtronic and the Abbott Diabetes Care are not involved in carrying out the trial, data analysis, data management and publication.

Competing interests None declared.

Patient consent for publication Not required.

Provenance and peer review Not commissioned; externally peer reviewed.

Supplemental material This content has been supplied by the author(s). It has not been vetted by BMJ Publishing Group Limited (BMJ) and may not have been peer-reviewed. Any opinions or recommendations discussed are solely those of the author(s) and are not endorsed by BMJ. BMJ disclaims all liability and responsibility arising from any reliance placed on the content. Where the content includes any translated material, BMJ does not warrant the accuracy and reliability of the translations (including but not limited to local regulations, clinical guidelines, terminology, drug names and drug dosages), and is not responsible for any error and/or omissions arising from translation and adaptation or otherwise.

Open access This is an open access article distributed in accordance with the Creative Commons Attribution Non Commercial (CC BY-NC 4.0) license, which permits others to distribute, remix, adapt, build upon this work non-commercially, and license their derivative works on different terms, provided the original work is properly cited, appropriate credit is given, any changes made indicated, and the use is non-commercial. See: http://creativecommons.org/licenses/by-nc/4.0/.

ORCID iD

Yongwen Zhou http://orcid.org/0000-0002-0558-6712

\section{REFERENCES}

1 Nathan DM, Genuth S, Diabetes Control and Complications Trial Research Group. The effect of intensive treatment of diabetes on the development and progression of long-term complications in insulindependent diabetes mellitus. N Engl J Med 1993;329:977-86.

2 Miller KM, Foster NC, Beck RW, et al. Current state of type 1 diabetes treatment in the U.S.: updated data from the T1D exchange clinic registry. Diabetes Care 2015;38:971-8.

3 Eeg-Olofsson K, Cederholm J, Nilsson PM, et al. Glycemic and risk factor control in type 1 diabetes: results from 13,612 patients in a national diabetes register. Diabetes Care 2007;30:496-502.

4 McKnight JA, Wild SH, Lamb MJE, et al. Glycaemic control of Type 1 diabetes in clinical practice early in the 21st century: an international comparison. Diabet Med 2015;32:1036-50.

5 Liu L, Yang D, Zhang Y, et al. Glycaemic control and its associated factors in Chinese adults with type 1 diabetes mellitus. Diabetes Metab Res Rev 2015;31:803-10.

6 American Diabetes Association. Glycemic targets: standards of medical care in diabetes-2018. Diabetes Care 2018;41:S55-64.

7 Simmons JH, Chen V, Miller KM, et al. Differences in the management of type 1 diabetes among adults under excellent 
control compared with those under poor control in the T1D exchange clinic registry. Diabetes Care 2013;36:3573-7.

8 Tamborlane WV, Beck RW, Juvenile Diabetes Research Foundation Continuous Glucose Monitoring Study Group. Continuous glucose monitoring and intensive treatment of type 1 diabetes. $N$ Engl $J$ Med 2008;359:1464-76.

9 Polonsky WH, Hessler D, Ruedy KJ, et al. The impact of continuous glucose monitoring on markers of quality of life in adults with type 1 diabetes: further findings from the diamond randomized clinical trial. Diabetes Care 2017:40:736-41.

10 Lind M, Polonsky W, Hirsch IB, et al. Continuous glucose monitoring vs conventional therapy for glycemic control in adults with type 1 diabetes treated with multiple daily insulin injections: the gold randomized clinical trial. JAMA 2017;317:379-87.

11 , Bode B, Beck RW, Juvenile Diabetes Research Foundation Continuous Glucose Monitoring Study Group. Sustained benefit of continuous glucose monitoring on A1c, glucose profiles, and hypoglycemia in adults with type 1 diabetes. Diabetes Care 2009;32:2047-9.

12 Ólafsdóttir AF, Attvall S, Sandgren U, et al. A clinical trial of the accuracy and treatment experience of the flash glucose monitor FreeStyle Libre in adults with type 1 diabetes. Diabetes Technol Ther 2017:19:164-72.

$13 \mathrm{Ji}$ L, Guo X, Guo L, et al. A multicenter evaluation of the performance and usability of a novel glucose monitoring system in Chinese adults with diabetes. J Diabetes Sci Technol 2017;11:290-5.

14 Fokkert MJ, van Dijk PR, Edens MA, et al. Performance of the FreeStyle Libre flash glucose monitoring system in patients with type 1 and 2 diabetes mellitus. BMJ Open Diabetes Res Care 2017;5:e000320-e.

15 Garg SK, Akturk HK. Flash glucose monitoring: the future is here. Diabetes Technol Ther 2017;19:S1-3.

16 Herman A, Aerts O, Baeck M, et al. Allergic contact dermatitis caused by isobornyl acrylate in Freestyle $₫$ Libre, a newly introduced glucose sensor. Contact Dermatitis 2017;77:367-73.

17 Heinemann L, Freckmann G, Versus FGM CGM. CGM versus FGM; or, continuous glucose monitoring is not flash glucose monitoring. $J$ Diabetes Sci Technol 2015;9:947-50.

18 Bolinder J, Antuna R, Geelhoed-Duijvestijn P, et al. Novel glucosesensing technology and hypoglycaemia in type 1 diabetes: a multicentre, non-masked, randomised controlled trial. Lancet 2016;388:2254-63.

19 Reddy M, Jugnee N, El Laboudi A, et al. A randomized controlled pilot study of continuous glucose monitoring and flash glucose monitoring in people with type 1 diabetes and impaired awareness of hypoglycaemia. Diabet Med 2018;35:483-90.

20 Feig DS, Donovan LE, Corcoy R, et al. Continuous glucose monitoring in pregnant women with type 1 diabetes (CONCEPTT): a multicentre international randomised controlled trial. Lancet 2017;390:2347-59.
21 Boucher SE, Gray AR, Wiltshire EJ, et al. Effect of 6 months of flash glucose monitoring in youth with type 1 diabetes and highrisk glycemic control: a randomized controlled trial. Diabetes Care 2020;43:dc200613.

22 Secher AL, Pedersen-Bjergaard U, Svendsen OL, et al. Study protocol for optimising glycaemic control in type 1 diabetes treated with multiple daily insulin injections: intermittently scanned continuous glucose monitoring, carbohydrate counting with automated bolus calculation, or both? a randomised controlled trial. BMJ Open 2020;10:e036474.

23 Alberti KG, Zimmet PZ. Definition, diagnosis and classification of diabetes mellitus and its complications. Part 1: diagnosis and classification of diabetes mellitus provisional report of a who consultation. Diabet Med 1998;15:539-53.

24 Expert Committee on the Diagnosis and Classification of Diabetes Mellitus. Report of the expert Committee on the diagnosis and classification of diabetes mellitus. Diabetes Care 2003;26:s5-20.

25 Qing Y, Xueqing L. Reliability and validity of the diabetes distress scale. J Nurs 2010;17:8-10.

26 MU C, Qi B, QIULING L. The reliability and validity of Chinese version of hypoglycemia fear survey II-Worry scale (CHFSII-WS) in type 2 diabetes mellitus. Chinese J Pract Nurs 2015;31:198-201.

27 Yabin X, Aixia M. Study on reliability and validity of Chinese version of EQ-5D-5L. Shanghai Med Pharma J 2013;9:40-3.

28 Danne T, Nimri R, Battelino T, et al. International consensus on use of continuous glucose monitoring. Diabetes Care 2017;40:1631-40.

29 Asarani NAM, Reynolds AN, Boucher SE, et al. Cutaneous complications with continuous or flash glucose monitoring use: systematic review of trials and observational studies. J Diabetes Sci Technol 2020;14:328-37.

30 Beck RW, Riddlesworth T, Ruedy K, et al. Effect of continuous glucose monitoring on glycemic control in adults with type 1 diabetes using insulin injections: the diamond randomized clinical trial. JAMA 2017;317:371-8.

31 Ruedy KJ, Parkin CG, Riddlesworth TD, et al. Continuous glucose monitoring in older adults with type 1 and type 2 diabetes using multiple daily injections of insulin: results from the diamond trial. $J$ Diabetes Sci Technol 2017;11:1138-46.

32 Mauras N, Beck R, Xing D, et al. A randomized clinical trial to assess the efficacy and safety of real-time continuous glucose monitoring in the management of type 1 diabetes in young children aged 4 to $<10$ years. Diabetes Care 2012;35:204-10.

33 Tsalikian E, Fox L, Weinzimer S, et al. Feasibility of prolonged continuous glucose monitoring in toddlers with type 1 diabetes. Pediatr Diabetes 2012;13:294-300.

34 Evans M, Welsh Z, Ells S, et al. The impact of flash glucose monitoring on glycaemic control as measured by HbA1c: a metaanalysis of clinical trials and real-world observational studies. Diabetes Ther 2020;11:83-95. 